\title{
ON MODELLING BEACH PROFILE EVOLUTION
}

\author{
Harshinie Karunarathna ${ }^{1}$ Dominic Reeve ${ }^{2}$ and Mark Spivack ${ }^{3}$
}

\begin{abstract}
Behaviour-oriented beach evolution models are normally applied in a prognostic fashion, with model parameters and boundary conditions estimated from previous experience, other forecasts or from historic measurements. Here, we use observations of beach profiles to solve a cross-shore beach profile evolution equation in an inverse manner to determine key model parameters, cross-shore sediment diffusion coefficient and a time-varying source function. The data used to demonstrate the method are from Christchurch Bay in Dorset, United Kingdom. It was found that there is a significant contribution from diffusive processes to the morphodynamic variability of the beach profiles and that the development and disappearance of cross-shore coastal features such as upper beach berms and inter- and sub-tidal bars are well captured by the time-varying source function in the governing equation.
\end{abstract}

Keywords: coastal morphology, beach profiles, inverse method, behaviour-oriented models

\section{INTRODUCTION}

Morphodynamic predictions of coastal systems are based on two modelling approaches (De Vriend, et al., 1993; De Vriend, 2003). The first approach is the use of process models based on two or three dimensional hydrodynamic models combined with sediment transport and morphodynamic modules (van Rijn et al., 2003; Roelvink et al., 2001). These models are a valuable tool for assessing local, short-term morphodynamic changes in a beach, but have inherent limitations due to the lack of knowledge of sediment transport processes and their linkage to hydrodynamics. Uncertainties in the predictions are amplified by treating sediment with a range of grain sizes. Further, numerical predictions can exhibit great sensitivity to the initial conditions. This is due not just to the accumulation of numerical rounding errors in the computations required to solve the equations but also due to nonlinearity of many coastal systems that may induce chaotic behaviour. The second group of models has been termed 'behaviour-oriented models'. These models are designed to overcome the difficulties arising out of application of process based modelling (Cowell, 1992, 1994; Dean, 1991; Stive and de Vriend, 1995; Reeve and Fleming 1997). The aim of behaviour-oriented models is to reproduce the qualitative behaviour of beach morphology using a simplified governing equation, parameterising only the key processes. The governing equations are rarely derived from first principles; rather, they are defined along the lines of physical arguments. This, and the parameterisation of processes, are both the strength and potential weakness of such methods.

Diffusion type formulations have been used in the past to model long term coastal and estuarine morphodynamic behaviour. It is important to note that this type of equations that have been applied to coastal morphology have not derived rigorously from basic process equations but are selected because their solutions qualitatively exhibit the behaviour of the application (Pelnard-Considère 1956; Stive and De Vriend, 1995; Reeve and Spivack, 1994, 2000; Hansen, et al., 2003; Karunarathna et al., 2008). The success of these models depends on the identification of fundamental parameters as the space and time varying coefficients of a simplified dynamic equation. In the application of a diffusion type model to beach profile change, collective changes to beach profile morphology including development, disappearance and evolution of near-shore morphological features and flattening and steepening of the profile, which are driven by external forcing are all included in a source function, which is reproduced based on field evidence.

We present a technique for the determination and recovery of the diffusion coefficient and an unknown source function in an advection-diffusion type governing equation for long-term beach profile evolution in this paper. The diffusion coefficient is derived as a problem of error minimisation and the source function is recovered as the solution of an inverse problem, using measurements of historic cross-shore beach profiles.

\section{FORMULATION OF THE MODEL}

Following the approach suggested by Stive and de Vriend (1995) we take a form of 1-D advectiondiffusion equation relative to a fixed reference level as the governing equation for the evolution of cross-shore beach profiles:

\footnotetext{
${ }^{1}$ Department of Civil Engineering, University of Glasgow, Glasgow G12 8LT, UK

2 School of Marine Science and Engineering, University of Plymouth, Plymouth PL4 8AA, UK

${ }^{3}$ Department of Applied Mathematics and Theoretical Physics, University of Cambridge, Cambridge CB3 OWA, UK
} 


$$
\frac{\partial h(x, t)}{\partial t}=\frac{\partial}{\partial x}\left(K(x) \frac{\partial h(x, t)}{\partial x}\right)+S(x, t)
$$

in which $h(x, t)$ is the cross-shore beach profile depth measured relative to a fixed reference line, $x$ is the cross-shore position, $K(x, t)$ and $S(x, t)$ are space and time dependent sediment diffusion coefficient and an external source function respectively. Figure 1 shows the schematics of the model.

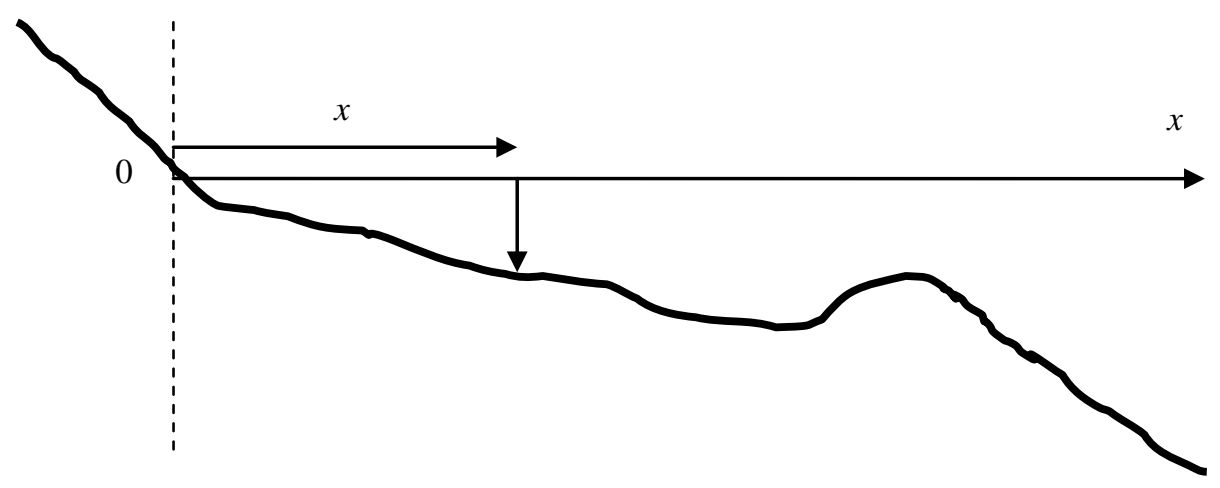

Figure 1 - Schematics of the cross-shore profile evolution model.

There are two unknowns to be resolved in the governing equation, Equation (1): the sediment diffusion coefficient $K(x, t)$ and the time and space varying source function $S(x, t)$. To solve equation (1) to forecast future evolution of cross-shore beach profiles, these two unknowns should be found. Finding suitable values for the diffusion coefficient and the source function is the key element to the success of the model.

The spatial variation of the diffusion coefficient allows us to represent the variation of morphological time scale with cross-shore position. All information about the typical site climate, sediment characteristics and short term dynamics are assumed to be summarised in $K$. All other natural inputs from climate change and human induced inputs are included in the source function $S$.

Next we perform a 'Reynolds' expansion', writing the profile depth $h$, the diffusion coefficient $K$ and the source function $S$ as the sum of their time averaged value and a time varying component as follows:

$$
\begin{aligned}
& h(x, t)=\bar{h}(x)+h^{\prime}(x, t) \\
& K(x, t)=\bar{K}(x)+K^{\prime}(x, t) \\
& S(x, t)=\bar{S}(x)+S^{\prime}(x, t)
\end{aligned}
$$

where the over-bar denotes the time averaged components and a prime denotes the time varying residuals.

Then, the equation (1) can be re-written as

$$
\frac{\partial\left[\bar{h}(x)+h^{\prime}(x, t)\right]}{\partial t}=\frac{\partial}{\partial x}\left(\left[\bar{K}(x)+K^{\prime}(x, t)\right] \frac{\partial\left[\bar{h}(x)+h^{\prime}(x, t)\right]}{\partial x}\right)+\bar{S}(x)+S^{\prime}(x, t)
$$

or,

$$
\frac{\partial\left[\bar{h}(x)+h^{\prime}(x, t)\right]}{\partial t}=\frac{\partial}{\partial x}\left(\bar{K}(x) \frac{\partial h(x, t)}{\partial x}\right)+\bar{G}(x)+G^{\prime}(x, t)
$$


where we have written

$$
G(x, t)=\frac{\partial}{\partial x}\left(K(x, t) \frac{\partial[h(x, t)]}{\partial x}\right)+S(x, t)
$$

$G$ is the new source function.

We assume that the time average is taken over a sufficiently long period that for any variable $x$, $\overline{x^{\prime}}=0, \partial \bar{x} / \partial t \approx 0$ and that to a first approximation $\bar{S} \approx 0$.

Then,

$$
\bar{G}(x)=\overline{\frac{\partial}{\partial x}\left(K(x, t) \frac{\partial[h(x, t)]}{\partial x}\right)}
$$

Taking the time average of Equation (6) gives:

$$
0=\frac{\partial}{\partial x}\left(\bar{K}(x) \frac{\partial \bar{h}(x, t)}{\partial x}\right)+\bar{G}(x, t)
$$

In an analogy to the Reynolds' stresses of turbulent fluid flow, $\bar{G}(x)$ may be considered to be a turbulent morphodynamic stress. As a first order approximation we take these stresses to be zero.

Equation (9) is then solved for time averaged component of the beach profile,

$$
\frac{\partial}{\partial x}\left(\bar{K}(x) \frac{\partial \bar{h}(x)}{\partial x}\right)=0
$$

The solution of which gives

$$
\bar{K}(x)=\frac{\alpha}{\left(\frac{\partial \bar{h}(x)}{\partial x}\right)}
$$

where $\alpha$ is a constant of integration. $\left(\frac{\partial \bar{h}(x)}{\partial x}\right)$ is the gradient of the mean cross-shore beach profile, which may be calculated from the measurements of cross-shore beach profiles, and must not be equal to zero anywhere in the range of $x$ considered.

The physical interpretation of Equation (11) is quite straightforward. To maintain a steep beach the mean sediment diffusion coefficient must be small. Conversely, a large value of the diffusion coefficient corresponds to a gently sloping beach. This accords with the observation that gravel beaches that comprised of coarse sediment are generally steep while sandy beaches, composed of highly mobile fine sediment, adopt gentler slopes.

\section{Determination of time averaged diffusion coefficient}

In any application of this methodology, it is understood that a time history of cross-shore beach profile measurements is available. From these, it will be possible to estimate the mean beach profile and hence it's gradient. This can be used in Equation (11) as a known quantity. However, there are two 
unknowns: $\bar{K}(x)$ and $\alpha$. To solve Equation (11) for $\bar{K}(x)$ a value for $\alpha$ must be specified. Rather than selecting a single value we adopt a procedure similar to that used by Reeve \& Fleming (1997):

Let $x_{i}(i=1,2, \ldots . . N)$ denote the $x$-coordinates for which the average beach profile depth $\bar{h}(x)$ is known and let the times for which beach profile depths are known be denoted by $t_{\mathrm{j}}(j=1,2, \ldots \ldots . M)$. Then, a sequence of discrete values of $\alpha, \alpha_{k}(k=1,2, \ldots . L)$ were defined and a corresponding sequence of solutions for $\bar{K}(x), \bar{K}(x)_{k}(k=1,2, \ldots \ldots . L)$, were calculated. Following that, for each time interval $t_{j}$ to $t_{j+1}$ with $1<j<\mathrm{M}$, predictions of the beach profile for $t_{j+1}$ were made using $\bar{K}(x)_{k}$ in place of $K$ in Equation (1) without the source function as follows:

$$
h(x, t+\tau)_{C}=h(x, t)+\frac{\tau}{\Delta x}\left[\bar{K}(x)_{i+1}\left(\frac{\partial h(x, t)}{\partial x}\right)_{i+1}-\bar{K}(x)_{i-1}\left(\frac{\partial h(x, t)}{\partial x}\right)_{i-1}\right]
$$

The discrepancy between predictions and known measured beach profiles for each time interval $\varepsilon_{\mathrm{j}}$ was calculated as the root mean square difference between the predicted and measured beach profile depth:

$$
\varepsilon_{j}=\sqrt{\frac{\sum_{i=1}^{N}\left[h\left(x_{i}, t_{j+1}\right)_{M}-h\left(x_{i}, t_{j+1}\right)_{C}\right]^{2}}{N}}
$$

Where $h\left(x_{i}, t_{j+1}\right)_{M}$ and $h\left(x_{i}, t_{j+1}\right)_{C}$ are the measured and predicted values of profile depth at the next time step respectively.

The discrete values of $\varepsilon_{j}$ can be considered to define $\varepsilon_{j}$ as a continuous function of $\alpha$. The value of $\alpha$ which corresponds to the minimum of this function was taken as the best fit value of $\bar{K}(x)$. Physically, this process corresponds to selecting the mean diffusion coefficient so that it explains, as much of the observed change, as possible. However, any morphological change not consistent with Equation (12) will appear as part of the source function.

\section{Recovery of the space- and time-varying source function}

The time-averaged diffusion coefficient $\bar{K}(x)$ is then used in Equation (7) to derive the source function $G(x, t)$ as an inverse problem as follows.

Re-writing the Equation (6) in operator notation gives

$$
h_{t}=D h+G
$$

where the operator $D=\frac{\partial}{\partial x}\left(\bar{K}(x) \frac{\partial h}{\partial x}\right)$.

Spivack and Reeve $(1999,2000)$ showed that if the time variation of $G(x, t)$ is weak enough to be neglected over one time step $\tau$ then the formal solution of Equation (14) can be written as

$$
h\left(x_{i}, t_{j+1}\right) \cong(\exp (D \tau)-1) D^{-1} G+\exp (D \tau) h\left(x_{i}, t_{j}\right)
$$

where $\tau=t_{j+1}-t_{j}$. 
The exponential terms are differential operators acting on the functions $G(x, t)$ and $h(x, t)$. It has been assumed that, for simplicity, the values of $h(x, t)$ are given at uniform intervals at a series of time steps $t_{j}$ where $x_{i}$ is evenly spaced.

Using first order approximation of exponential terms, an expression for the source function $G(x, t)$ is found as

$$
G(x, t)=\frac{1}{\tau}[h(t+\tau)-\exp (D \tau) h(t)]
$$

Equation (16) gives an explicit expression for the unknown source function. Given the data for the function $h(x, t)$ and pre-determined time mean diffusion coefficient, source function $G$ can be recovered from Equation (16).

\section{FIELD SITE AND BEACH PROFILE DATA}

The data used to derive the diffusion coefficient and the source function is a set of historic crossshore beach profile surveys measured at Milford-on-Sea beach, Christchurch Bay, United Kingdom. The profile data was provided by the Channel Coastal Observatory, UK. Figure 2 shows a map of the beach and its location in the UK. The bay, located in the south coast of the UK, is about $4 \mathrm{~km}$ long and bounded by Hurst Spit to the east and Hengistbury Head to the west. Milford-on-Sea is a sand-gravel mixed beach with multiple shore parallel bars and is rapidly evolving in time. The upper and middle beach is mostly shingle while lower inter-tidal and sub-tidal areas including sub-tidal bars are mostly sand. A part of the beach is backed by soft, eroding cliffs and a part is protected by coastal defence structures including seawalls and shore normal groynes.

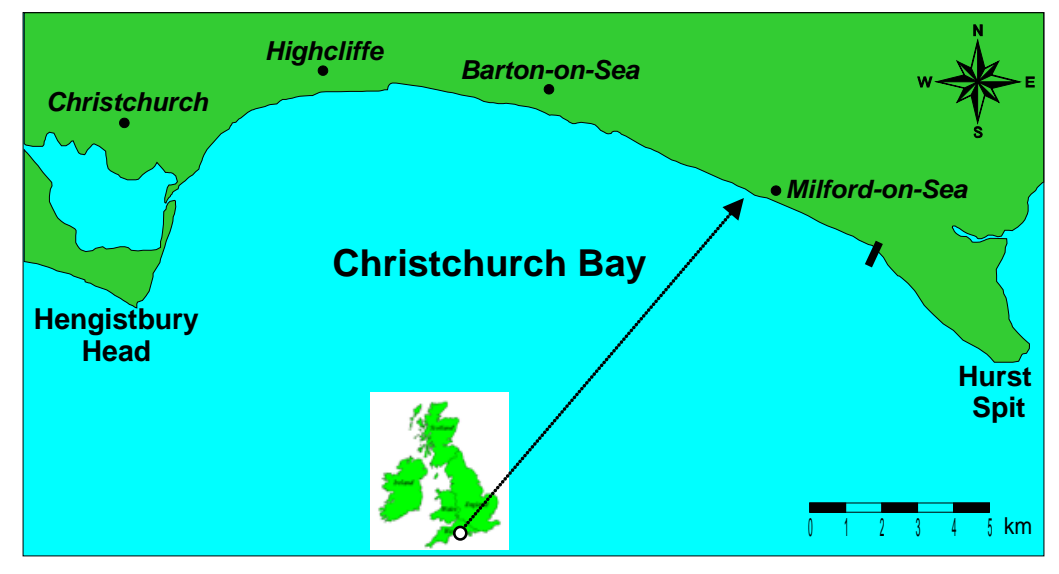

Figure 2 - Christchurch Bay and its location in the United Kingdom.

Cross-shore beach profiles at Christchurch Bay have been surveyed since 1987 at numerous transects along the entire coastline. Beach profile surveys at cross-shore transect $5 f 00070$ (see Figure 1 for survey location) were selected to demonstrate the methodology described in this paper, as it has the best data coverage and a good cross-shore extend.

Measured cross-shore profiles at $5 f 00070$ are shown in Figure 3. It was observed that the overall beach elevation at this location varied between 1-2 m. The still water shoreline moved 10-15 m shoreward or seaward accordingly. An upper beach berm seems to appear and disappear from time to time. A shallow trough and an alongshore bar was observed in some surveys. The sub-tidal area of the beach is much gentler than the steep upper beach face and the inter-tidal beach.

Some of the beach profile surveys cover the entire profile from the upper beach to the sub-tidal terrace while some surveys were incomplete and cover only the upper and inter-tidal beach. Also, survey intervals were not uniform and profile depths were not been surveyed at uniform spatial intervals. To maximise the number of usable cross-shore surveys, the beach profiles were first curtailed at $30 \mathrm{~m}$ cross-shore chainage from the cliff foot. Then, the time-mean profile was calculated using all measured profile data. The time-mean profile was fairly uniform with a slight reduction in gradient towards the sub-tidal beach. The profile data were then interpolated on to uniform spatial intervals $(0.5 \mathrm{~m})$ along the profiles and temporal intervals (90 days) using Akima interpolation routine. This is a continuously differentiable sub-spline interpolation built from piece-wise third order polynomials. This 
was necessary as the methodology derived to recover the source function demands uniform spatial and temporal intervals of beach profile measurements.

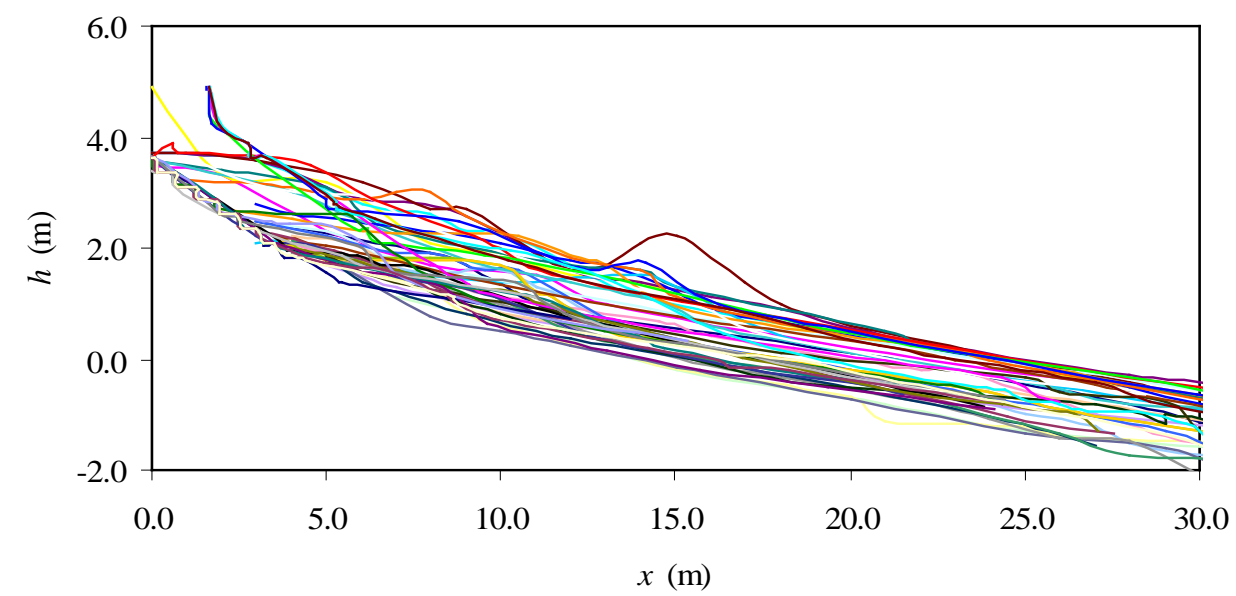

Figure 3 - Measured cross-shore beach profiles at transect 5 f001070 at Milford-on-Sea from 1987 to 2005. Profiles extend from the top of the dune to the mean low water level.

\section{RESULTS}

Based on sediment diffusion coefficients for different sediment sizes reported in literature (Huthnance 1982a, 1982b) first, we estimated an initial value of $10 \mathrm{~m}^{2} / \mathrm{yr}\left(0.027 \mathrm{~m}^{2} /\right.$ day) for the mean sediment diffusion coefficient $\bar{K}$ at Milford-on-Sea beach. The (spatial) mean of the beach profile gradient $\left(\frac{\partial \bar{h}(x)}{\partial x}\right)$ was determined from the cross-shore profiles measured at transect $5 f 00070$ as 0.11 , leading to an initial estimate of $\alpha \approx 3 \times 10^{-3} \mathrm{~m}^{2} /$ day. Based on this initial estimate for $\alpha, \varepsilon_{j}$ were calculated, following the method described in the previous section, using the measured values of $h(x, t)$ at transect $5 \mathrm{f} 00070$, for a range of $\alpha$ values varying from $10^{-4}$ to $10^{-2} \mathrm{~m}^{2} /$ day. Values of $\varepsilon_{j}$ varied between 0.05 and 0.08 . The minimum $\varepsilon_{j}$ was found at $\alpha=7 \times 10^{-4}$.

Following the method described in the previous section, the mean diffusion coefficient $\bar{K}$ across the transect 5f00070, was then calculated using the $\alpha$ value determined above. Figure 4 shows the cross-shore variation of $\bar{K}$. Mean cross-shore beach profile is also drawn in the figure. The mean sediment diffusion coefficient gradually increases in the offshore direction. This is as might be expected as the beach sediment varies from coarse shingle in the upper and middle beach to sand in the lower sub-tidal areas of the profile.

The spatially varying mean diffusion coefficient is then used in recovering the source function in the advection-diffusion profile evolution equation. The source function was computed using Equation (16), as a solution of an inverse problem using measured profile survey data described above.

To investigate the temporal variation of the source function in greater detail, time histories of it are drawn in Figure 5. Positive values correspond to beach accretion while negative values correspond to erosion. According to Figure 5, the source function captures alternate erosion and accretion of the cross-shore profile at seasonal/yearly timescales. In the period between 1996 and 1999, the source function show considerably low variability compared to the rest of the time, indicating a significant deviation from the general trend. It has been reported that substantial beach refilling had been taken place during this period (SCOPAC, 2003), which may have contributed to low profile variability. 


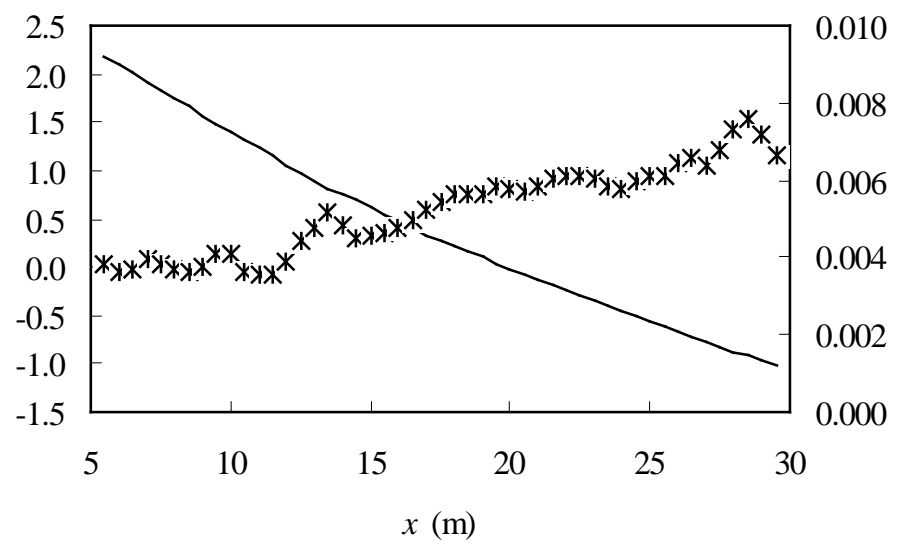

Figure 4 - Cross-shore variation of mean sediment diffusion coefficient $\bar{K}$ and mean profile depth $\bar{h}$ (full line) at transect $5 f 00070$.

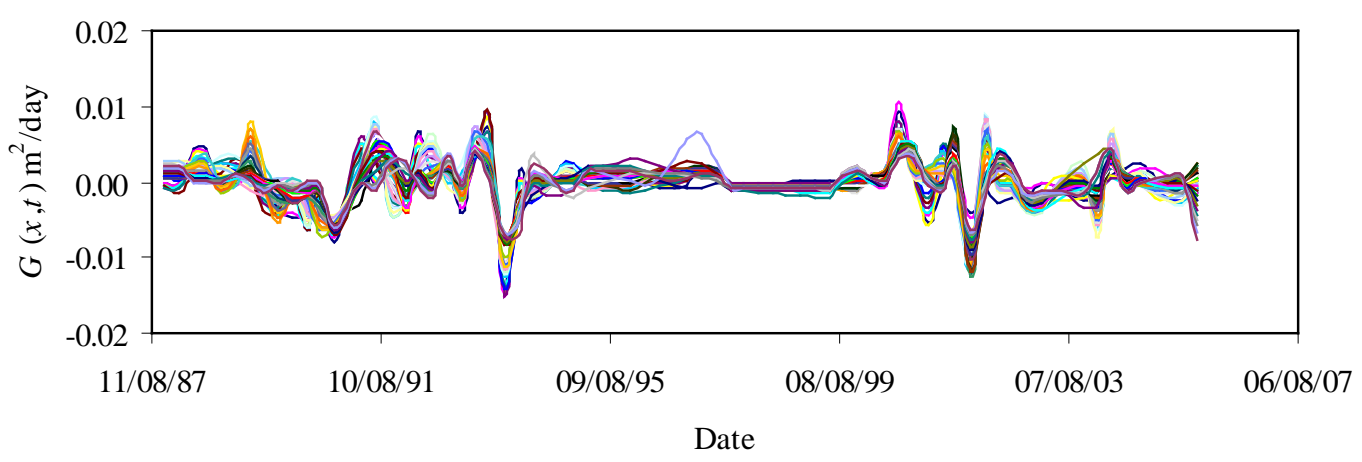

Figure 5 - Time variation of source function $G(x, t)$ at $5 f 00070$ (each line corresponds to a different cross-shore location)

Investigation of the entire set of source functions reveals that broad features are apparent in the source functions. A significant structure is persistent throughout the results. Evolution of profile features such as upper shore face berms and bars are persistently visible. Positive source functions indicate net accretion of the profile over the period concerned, faster than predicted by the process of large scale diffusion. Negative source functions indicate net profile erosion. Furthermore, all the source functions tend to zero in the offshore direction, indicating a gradual convergence to a depth of closure, or point at which zero net cross-shore transport occurs.

Figure 6 shows two selected source functions and the consecutive beach profiles used in recovering them. In these figures, the primary vertical axis corresponds to the cross-shore profile depth and the secondary vertical axis corresponds to the source function. It is apparent from Figure 6 that the source functions derived from a particular survey interval have significant differences from the corresponding beach profile change during that interval. The source functions are not simply a volume difference between two successive surveys. They represent the changes that have occurred relative to the (time) mean beach profile. The cross-shore shape of the source functions exhibits variation with time (not shown), reflecting the seasonal variation in wave energy reaching the beach. 




$X(\mathrm{~m})$

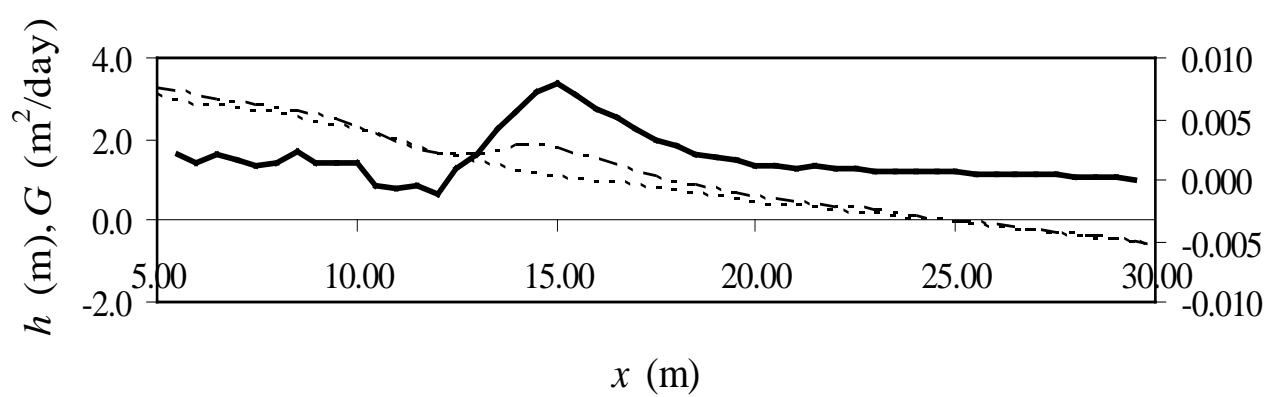

Figure 6- Source function ( - ) and corresponding beach profiles ( $\cdots \cdots \cdot$.... initial profile, $\quad-\cdot-$ final profile).

\section{CONCLUSIONS}

In this paper, a methodology for the recovery of the diffusion coefficient and the source function in an advection-diffusion type beach profile evolution model is described. This type of model is considered to be an extended behaviour oriented approach for predicting long term beach profile evolution. The governing equation isolates non-diffusive processes which drive sediment movement leading to profile evolution from the diffusive processes through an unknown source function. The source function then represents the aggregation of all non-diffusive phenomena which lead to morphodynamic evolution of the beach profile. The modelling approach determines a spatially varying diffusion coefficient and a source function using measured historic data on beach profile evolution.

The success of recovering a suitable diffusion coefficient and a source function using the present approach largely depends on the availability and accuracy of the measured cross-shore beach profile survey data. A good data set covering a considerable time period is needed to provide quantitative results. The implementation of the method is relatively straightforward and is computationally efficient.

The methodology has been demonstrated by obtaining solutions for the time-mean, space varying diffusion coefficient and time- and space-varying source function using historic data of cross-shore beach profiles at the Christchurch Bay, UK. It was found that there is a significant variation in the shape of the source function with time, which corresponds to the formation and disappearance of crossshore coastal features such as upper beach berms and inter- and sub-tidal bars.

As a final consideration we mention that the method described here may, possibly, form the basis of a method to forecast the beach shape. Such a method would rely on extrapolation of statistical analysis, and rely on past historical behaviour being a reliable indicator of future behaviour. The raw measurements could be extrapolated directly, of course. However, the level of noise makes this an uncertain procedure at best. Extrapolations of Fourier analysis, Empirical Orthogonal Function analysis, wavelet decomposition and so on succeed or fail through being able to identify strong underlying patterns of behavior such as trends and cycles. In a similar manner, identifying the diffusion coefficient and source functions in a simple beach model may provide some predictive capacity through extrapolation; and is the subject of ongoing research by the authors.

\section{ACKNOWLEDGMENTS}

The authors acknowledge the funding from EPSRC Grant No: EP/C005392/1. 


\section{REFERENCES}

Cowell, P.J., Roy, P.S. and Jones, R.A. 1992. Shoreface translation model: computer simulation of coastal sand body response to sea level rise, Mathmat. Compt. Simulation, Vol.33, 603-608.

Cowell, P.J., Roy, P.S. and Jones, R.A. 1994. Simulation of LSCB using a morphological behaviour model, Marine Geology, Vol. 126, pp.45-61.

Dean, R.G. 1991. Equilibrium beach profiles: Characteristics and applications, Journal of Coastal Research, Vol. 7(1), pp. 53-84.

De Vriend, H.J., Capobianco, M., Chester, T., Swart, H.E., Latteux, B. and Stive, M. 1993. Approaches t o long term modelling of coastal morphology: a review, Coastal Engineering, Vol. 21, pp. 225-269.

De Vriend, H.J. 2003. On the prediction of aggregate scale coastal evolution, J. Coastal Research, Vol. 19(4), pp. 757-759.

Hansen, H., Arrninkhof, S., Capobianco, M., Mimenez, J.A., Karson, M., Nicholls, R.J., Plant, N.G., Southgate, H.N., Steetzel, H.J., Stive, M.J.F. and De Vriend, H.J. 2003. Modelling of coastal evolution on early and decadal time scales, J. of Coastal Research, Vol. 19(4).

Huthnance, J.M. 1982a. On one mechanism forming linear sand banks, Estuarine Coastal and Shelf Sci. 14, 79-99.

Huthnance, J.M. 1982b. On the formation of sand banks of finite extent, Estuarine Coastal and Shelf Sci. 15, 277-299.

Karunarathna, H., Reeve, D.E. and Spivack, M. 2008. Long term morphodynamic evolution of estuaries: An inverse problem, Estuarine Coastal and Shelf Science, Vol. 77, pp. 386-395.

Pelnard-Considere, R. 1956. Essi de theorie de l'evolution des formes de ravage en plages de sables et de galets, Societe Hydrotechnique de France, IV'eme Journee de L'Hydraulique Question III, Rapport 1, 74-1-10.

Reeve D.E. and Fleming, C.A. 1997. A statistical-dynamical method for predicting long term coastal evolution, Coastal Engineering, Vol. 30, pp. 259-280.

Reeve, D.E. and Spivack, M. 1994. Determination of a source term in the linear diffusion equation, Inverse Problems, 10, pp. 1335-1344.

Reeve, D.E. and Spivack, M. 2000. Inverse methods in coastal morphology, Proc. Int. Conf. Coastal Eng., Sydney, Australia, pp. 3409-3418.

Roelvink, D., Jeuken, M.C.J.L., vanHolland, G., Aarninkhof, S.G.L. and Stam, J.M.T. 2001. Long term process based modelling of complex areas, Coastal Dynamics, Lund, Sweden, pp.383-392.

Standing Conference on problems Associated with the Coastline (SCOPAC). 2003. Sediment Transport Study - from Hengistbury Head to Hurst Spit.

Spivack, M., Reeve, D.E. 1999. Recovery of a variable coefficient in a coastal evolution equation. J. Comp. Phys. 151, 585-596.

Spivack, M. and Reeve, D.E. 2000. Source reconstruction in a coastal evolution equation, $J$. Computational Physics, 161, pp.169-181.

Stive, M.J.F. and De Vriend, H.J. 1995. Modelling shore-face profile evolution, Marine Geology, Vol.126, pp.235-248.

Van Rijn, L.C., Walstra, D.J.R., Grasmeijer, J., Sutherland, J., Pan, S. and Sierra, J.P. 2003. The predictability of cross-shore bed evolution of sandy beaches at the time scale of storms and seasons using process based profile models, Coastal Engineering, Vol. 47, pp. 295-327. 